\title{
G protein-coupled receptor 21 deletion improves insulin sensitivity in diet-induced obese mice
}

\author{
Olivia Osborn, ${ }^{1}$ Da Young Oh, ${ }^{1}$ Joanne McNelis, ${ }^{1}$ Manuel Sanchez-Alavez, ${ }^{2}$ Saswata Talukdar, ${ }^{1}$ \\ Min Lu, ${ }^{1}$ PingPing Li, ${ }^{1}$ Lucinda Thiede, ${ }^{3}$ Hidetaka Morinaga, ${ }^{1}$ Jane J. Kim, ${ }^{4}$ Jan Heinrichsdorff, ${ }^{1}$ \\ Sarah Nalbandian, ${ }^{1}$ Jachelle M. Ofrecio, ${ }^{1}$ Miriam Scadeng, ${ }^{5}$ Simon Schenk, ${ }^{6}$ John Hadcock, ${ }^{3}$ \\ Tamas Bartfai, ${ }^{2}$ and Jerrold M. Olefsky ${ }^{1}$
}

\begin{abstract}
${ }^{1}$ Division of Endocrinology and Metabolism, Department of Medicine, UCSD, La Jolla, California, USA. ${ }^{2}$ The Scripps Research Institute, La Jolla, California, USA. ${ }^{3}$ Cardiovascular, Metabolic, and Endocrine Diseases, Pfizer, Groton, Connecticut, USA. 4Department of Pediatrics, UCSD, and Rady Children's Hospital of San Diego, San Diego, California, USA. ${ }^{5}$ Department of Radiology, UCSD, San Diego, California, USA. ${ }^{6}$ Department of Orthopaedic Surgery, UCSD, La Jolla, California, USA.
\end{abstract}

\begin{abstract}
Obesity-induced inflammation is a key component of systemic insulin resistance, which is a hallmark of type 2 diabetes. A major driver of this inflammation/insulin resistance syndrome is the accumulation of proinflammatory macrophages in adipose tissue and liver. We found that the orphan GPCR Gpr21 was highly expressed in the hypothalamus and macrophages of mice and that whole-body $\mathrm{KO}$ of this receptor led to a robust improvement in glucose tolerance and systemic insulin sensitivity and a modest lean phenotype. The improvement in insulin sensitivity in the high-fat diet-fed (HFD-fed) Gpr21 KO mouse was traced to a marked reduction in tissue inflammation caused by decreased chemotaxis of $\mathrm{Gpr} 21 \mathrm{KO}$ macrophages into adipose tissue and liver. Furthermore, mice lacking macrophage expression of Gpr21 were protected from HFD-induced inflammation and displayed improved insulin sensitivity. Results of in vitro chemotaxis studies in human monocytes suggested that the defect in chemotaxis observed ex vivo and in vivo in mice is also translatable to humans. Cumulatively, our data indicate that GPR21 has a critical function in coordinating macrophage proinflammatory activity in the context of obesity-induced insulin resistance.
\end{abstract}

\section{Introduction}

Obesity-induced insulin resistance is a major factor in the etiology of type 2 diabetes, and the prevalence of these disorders is rising globally at epidemic rates. In recent years, chronic lowgrade inflammation has emerged as an important contributor to the development of insulin resistance (1-3). The inflammatory response triggered by obesity promotes infiltration of macrophages and other immune cells into adipose tissue and liver. For example, many studies have demonstrated increased transmigration and accumulation of adipose tissue macrophages (ATMs) in obesity $(4,5)$. The proinflammatory pathways of these ATMs are activated, leading to secretion of cytokines, which can directly impair insulin action. During the development of obesity, macrophages are also recruited to the liver (6), where activation of proinflammatory pathways results in decreased insulin-mediated suppression of hepatic glucose production (HGP) (7-10). The proinflammatory cytokines released by macrophages can have paracrine effects in the fat or liver and may also be transported via the systemic circulation, impairing insulin sensitivity in other tissues (2).

In a recent study to identify novel drug targets to treat obesity and type 2 diabetes, we characterized the expression profile of a subset of a hypothalamic cells that control core body temperature (CBT) (11). We hypothesized that receptors expressed in these temperature-sensing cells may play a role in regulation of CBT and energy homeostasis (12-14). Members of the GPCR superfamily, a major class of receptors that sense extracellular signals, are the

Conflict of interest: The authors have declared that no conflict of interest exists. Citation for this article: J Clin Invest. 2012;122(7):2444-2453. doi:10.1172/JCI61953. targets for a wide range of drugs (15). The orphan GPCR GPR21 was highly expressed in these temperature-sensing neurons, and we also noted that this receptor was highly expressed in macrophages. GPR21 couples to the Gq family of $\mathrm{G}$ proteins (16), but to our knowledge, there are no identified ligands, and its downstream signaling pathways remain to be determined.

Based on its tissue expression patterns, we investigated the role of GPR21 in obesity, insulin resistance, and energy homeostasis by generating a colony of mice deficient in Gpr21. We found that Gpr $21 \mathrm{KO}$ mice were protected from obesity-induced inflammation and insulin resistance, in parallel with reduced macrophage infiltration into adipose tissue and liver, with a marked reduction in inflammation in these tissues. Gpr $21 \mathrm{KO}$ mice also displayed a modest lean phenotype attributable to increased energy expenditure (EE).

\section{Results}

Gpr21 expression. Quantitative PCR (q-PCR) showed that Gpr21 mRNA was highly expressed in the brain, specifically in the preoptic area of the hypothalamus (Figure 1, A and B). Because Gpr21 was also highly expressed in BM and spleen (Figure 1A), we assessed the cellular source of Gpr21 in obese and lean adipose tissue. Cell fractionation experiments were performed to determine relative Gpr21 mRNA levels in both adipocytes and the stromal vascular fraction (SVF) of adipose tissue from lean and high-fat diet-fed (HFD-fed) obese mice. As shown in Figure 1C, Gpr21 mRNA was not detected in adipocytes or SVF from lean animals, but was highly induced in the SVF in obese mice. Thus, obesity induced Gpr21 in the macrophage-containing fraction of adipose tissue. To investigate whether Gpr21 is expressed in proinflamma- 
A

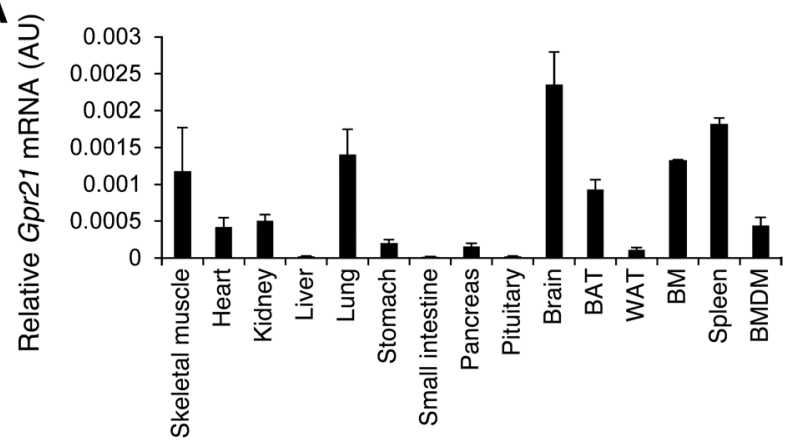

\section{Figure 1}

Quantification of Gpr21 expression. q-PCR analysis of WT Gpr21 mRNA expression in (A) various tissues and BM-derived macrophages (BMDM), (B) brain regions, (C) SVF and adipocytes of NC- and HFD-fed mice, and (D) M1-like (F4/80+CD11 b+CD11 $\mathrm{c}^{+}$) and $\mathrm{M} 2$-like $\left(\mathrm{F} 4 / 80^{+} \mathrm{CD} 11 \mathrm{~b}^{+} \mathrm{CD} 11 \mathrm{c}^{-}\right)$macrophages and T cells. WAT, white adipose tissue. Data (mean \pm SEM) are shown as fold induction of gene expression normalized to housekeeping gene ( $n=3$ per group).

In vivo metabolic studies in HFD-fed obese Gpr21 KO mice. HFD-fed Gpr21 KO mice gained BW in a manner similar to that of HFD-fed WT littermates and ate similar quantities of food (Figure 3, A and B, and Supplemental Figure 1B). Because the BW decreases were relatively small in Gpr $21 \mathrm{KO}$ mice, we were able to study BW-matched animals between the genotypes. GTTs in BW-matched animals showed markedly improved glucose tolerance with decreased insulinemia in Gpr21 KO mice (Figure 3, $\mathrm{C}$ and D). Hyperinsulinemic-euglycemic clamp studies revealed that Gpr21 deletion led to a significant increase in GIR

tory $\left(\mathrm{F} 4 / 80^{+} \mathrm{CD} 11 \mathrm{~b}^{+} \mathrm{CD} 11 \mathrm{c}^{+}\right.$; referred to herein as M1-like) or antiinflammatory (F4/80 ${ }^{+} \mathrm{CD} 11 \mathrm{~b}^{+} \mathrm{CD} 11 \mathrm{c}^{-}$; M2-like) macrophages, we sorted the SVF for these 3 markers using fluorescence-activated cell sorting (FACS). Gpr21 was highly expressed in M2-like macrophages, with lower expression in M1-like macrophages and no detectable expression in T lymphocytes (Figure 1D).

In vivo metabolic studies in Gpr21 KO mice fed normal chow (NC). The BW of NC-fed Gpr21 KO mice was significantly less than that of WT littermates $(P<0.05)$, despite similar food intake (Figure 2, A and B). Glucose tolerance tests (GTTs) showed improved glucose tolerance, with decreased insulin levels, in Gpr21 KO mice compared with WT littermates (Figure 2, C and D), indicative of enhanced insulin sensitivity. Consistent with this, Gpr21 $\mathrm{KO}$ mice exhibited greater responsiveness to insulin during insulin tolerance tests (ITTs; Figure 2E and Supplemental Figure 1A; supplemental material available online with this article; doi:10.1172/JCI61953DS1). This was confirmed by hyperinsulinemic-euglycemic clamp studies in NC-fed Gpr21 KO and WT littermates (Figure 2, F-J, and Supplemental Figure 2), which revealed that a significant increase in the glucose infusion rate (GIR) was required to maintain euglycemia in Gpr21 KO mice (Figure 2F). Gpr21 KO mice also exhibited decreased basal HGP and an increased ability of insulin to suppress HGP (Figure 2, $\mathrm{G}$ and $\mathrm{H}$ ), demonstrating enhanced hepatic insulin sensitivity. We also observed an increased insulin-stimulated glucose disposal rate (IS-GDR) in Gpr21 KO mice (Figure 2I), indicative of improved skeletal muscle insulin sensitivity. There was no difference in insulin-stimulated FFA suppression in Gpr21 KO compared with WT mice (Figure 2J and Supplemental Figure 2J). NCfed Gpr21 KO mice showed higher EE than did WT littermates, and also consumed significantly more $\mathrm{O}_{2}$ and exhaled more $\mathrm{CO}_{2}$ in the dark cycle (Supplemental Figure 3). required to maintain euglycemia (Figure 3E and Supplemental Figure 2). The increased ability of insulin to suppress HGP (Figure 3, F and G) demonstrated enhanced hepatic insulin sensitivity. These observed increases in GIR and HGP suppression in Gpr21 $\mathrm{KO}$ mice were also significant in BW-matched mice (Figure 3, E and $G$ ), which demonstrated that these changes were independent of BW differences between WT and Gpr21 KO mice. The increased IS-GDR observed was suggestive of improved skeletal muscle insulin sensitivity, but BW-matched Gpr21 KO mice did not show a significant increase (Figure $3 \mathrm{H}$ ), which indicates that BW differences contribute to enhanced skeletal muscle insulin sensitivity in Gpr21 $\mathrm{KO}$ mice. The ability of insulin to suppress FFA production also increased in Gpr21 KO mice, a difference that trended toward significance $(P<0.1$; Figure 3I and Supplemental Figure $2 \mathrm{~K})$, indicative of improved adipose tissue insulin sensitivity. However, this effect was not observed in BW-matched Gpr21 KO mice (Figure 3I). We next harvested adipose, liver, and muscle tissue for ex vivo measurements of insulin action. Insulin-stimulated Akt phosphorylation was increased in liver and adipose tissue in Gpr21 KO compared with WT mice, but no significant difference was observed in muscle (Supplemental Figure 4). Taken together, these data indicated that deletion of Gpr21 leads to improved insulin sensitivity in the liver, adipose tissue, and muscle; that decreased BW contributes to improvements in adipose and muscle insulin sensitivity; and that the substantially enhanced hepatic insulin action observed is independent of BW changes.

Gpr21 KO mice showed higher EE during HFD feeding than did WT littermates, predominantly during the dark cycle (Figure 3J). Gpr21 KO mice also consumed significantly more $\mathrm{O}_{2}$ and exhaled more $\mathrm{CO}_{2}$ in the dark cycle than did WT littermates (Figure 3, K and L). Gpr21 KO and WT mice were implanted with telemetry devices facilitating the measurement of movement 
A

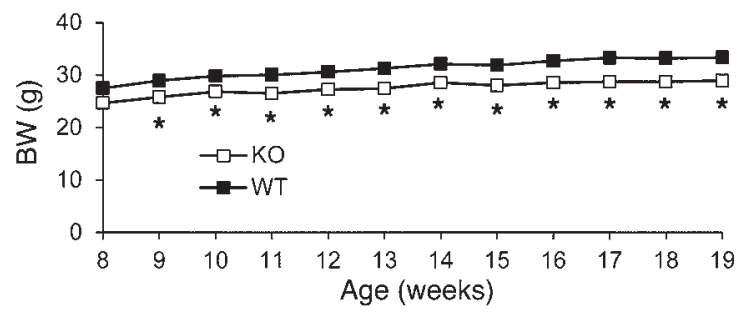

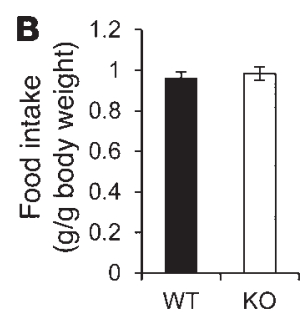

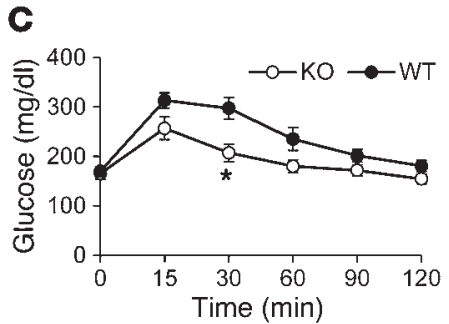

$\mathbf{F}$

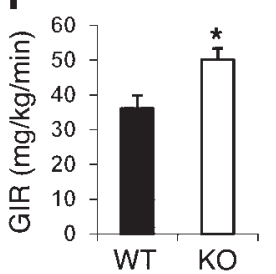

G

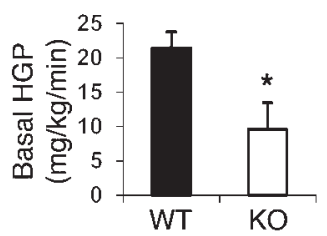

D

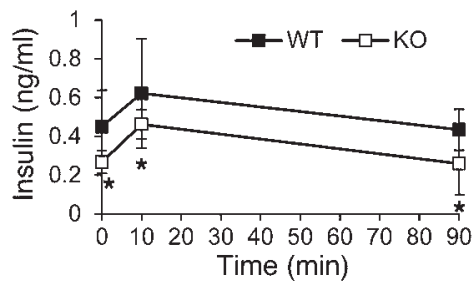

H

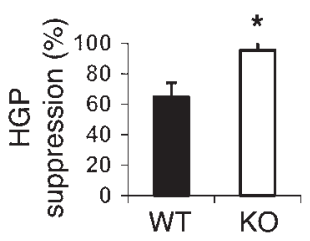

E

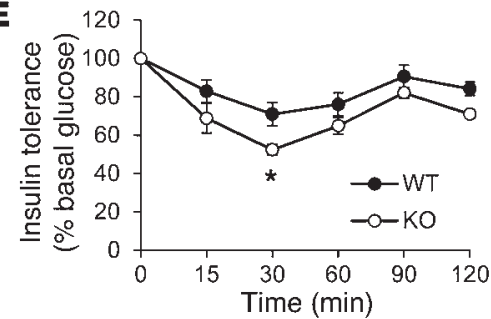

I

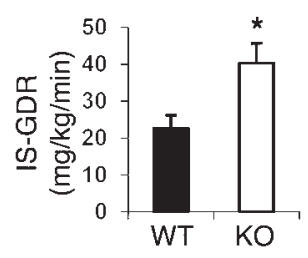

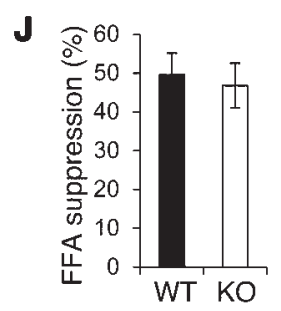

Figure 2

Effect of Gpr21 KO on BW gain, food intake, glucose tolerance, and insulin sensitivity. (A) BW gain and (B) weekly food intake of NC-fed Gpr21 KO and WT littermates ( $n=22-25$ per group). (C) i.p. GTT ( $1 \mathrm{~g} / \mathrm{kg}$ ) on Gpr21 KO and WT mice ( $n=8$ per group). (D) Insulin concentration at the indicated time points. (E) ITT on HFD-fed Gpr21 KO and WT mice ( $n=8$ per group), shown as percent change from basal blood glucose. (F-I) Hyperinsulinemic-euglycemic clamp study in Gpr21 KO and WT littermates ( $n=5-6$ per group). (F) GIR. (G) Basal HGP. (H) HGP suppression by insulin. (I) IS-GDR. (J) Insulin-induced FFA suppression. ${ }^{*} P<0.05$ vs. WT, repeated-measures 2 -way ANOVA (A and C-E) or Student's $t$ test (B and $\mathbf{F}-\mathbf{J})$.

and temperature at 15 -minute intervals for 24 hours. During the dark cycle, Gpr21 KO mice had higher CBT and were also more active than WT mice (Figure 3, M and N). When subjected to cold challenge $\left(4^{\circ} \mathrm{C}\right)$, Gpr $21 \mathrm{KO}$ mice were more tolerant to the cold and upregulated adaptive thermogenesis to increase their CBT to a greater extent than WT littermates over the first hour of cold exposure (Supplemental Figure 1C). Upon return to room temperature, Gpr21 KO mice recovered to normal basal temperature more rapidly than did WT littermates. After 14 weeks of HFD feeding, increased mRNA expression of the brown adipose tissue (BAT) gene markers Prdm16, Pgc1 $\alpha$, and Ucp1 was observed in Gpr21 KO mice (Figure 3O). Gpr21 KO mouse BAT increased in weight approximately $25 \%$ and also displayed a consistent decrease in proinflammatory gene expression compared with BAT of WT mice (Figure 3, P and Q).

Gpr21 KO mice display reduced inflammatory gene expression in liver and adipose tissue. In BW-matched cohorts, liver weight and hepatic TG and glycogen content were lower in Gpr21 KO mice (Figure 4, A-D). Proinflammatory gene expression was also lower in Gpr21 KO mouse liver (Figure 4E). Epididymal fat (eWAT) weight was similar in Gpr21 KO and WT mice (Figure 4F), and MRI of BWmatched mice revealed no differences in fat mass across compartments (Figure 4G). Interestingly, proinflammatory gene expression was decreased in eWAT from Gpr21 KO mice, whereas expression of antiinflammatory genes (e.g., Arg1) was increased (Figure 4H).
The modest lean phenotype observed in HFD-fed Gpr21 KO mice was due to decreased liver weight (in both non-BW-matched and BW-matched mice) and reduced lean mass, as there was no significant difference in eWAT weight between Gpr21 KO and WT mice in the non-BW-matched cohort (data not shown).

Gpr21 was expressed at a low level in the pancreas (Figure 1A), and no significant differences were observed in pancreatic weight, inflammatory gene expression, or $\beta$ cell area between Gpr21 KO and WT mice (Supplemental Figure 5).

ATMs in Gpr21 KO and WT mice. We conducted histological examination of ATMs from HFD-fed WT and Gpr21 KO mice. In eWAT of Gpr $21 \mathrm{KO}$ mice, we observed a substantial decrease in F4/80 immunostaining and fewer crown-like structures (Figure 5, A and B). Consistent with this, plasma levels of monocyte chemotactic protein-1 (MCP-1) were lower in BW-matched Gpr21 KO than WT mice (Figure 5C). To quantitate the proportion of M1-like macrophages within the adipose tissue, we isolated the SVF from NC- and HFD-fed Gpr21 KO and WT mice for FACS analysis. We observed a reduced percentage of the M1-like macrophages in Gpr21 KO mice in both NC and HFD states (Figure 5D).

We next tested the migratory capacity of i.p. macrophages from Gpr21 KO and WT littermates using an in vitro transwell chemotaxis assay. As shown in Figure 5E, macrophages from WT mice migrated rapidly toward conditioned media $(\mathrm{CM})$ harvested from 3T3L1 adipoctyes, whereas chemotaxis of Gpr21 KO i.p. macrophages was not 
A
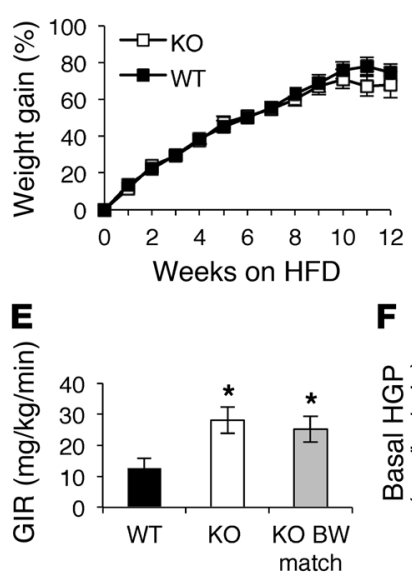

F
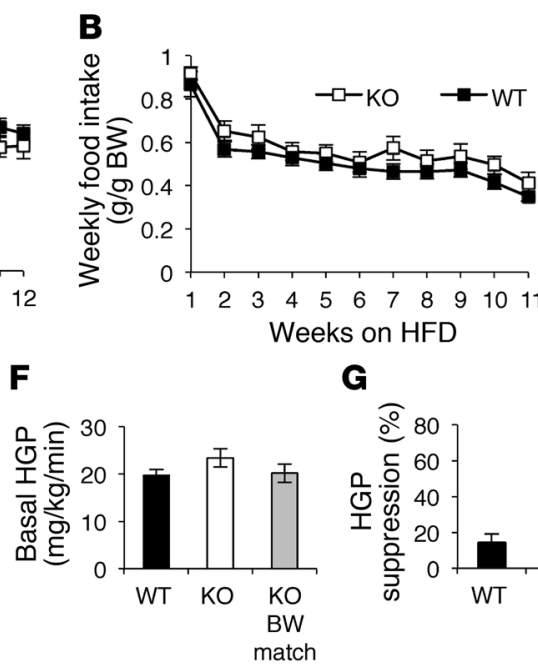

G

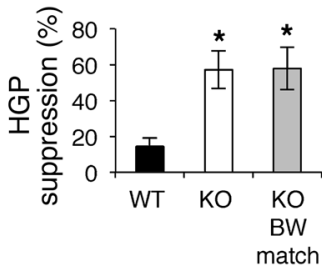

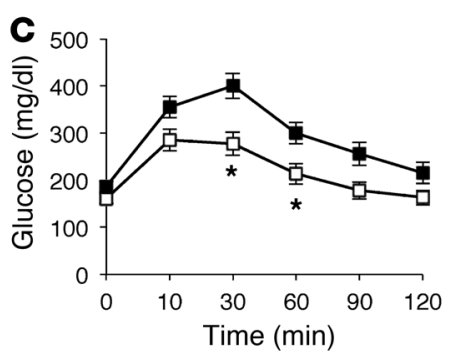

H

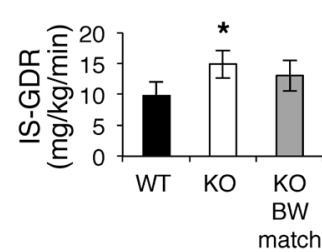

D

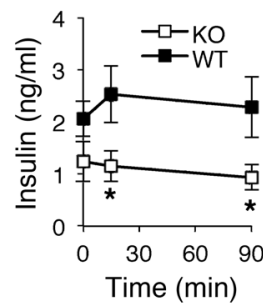

I

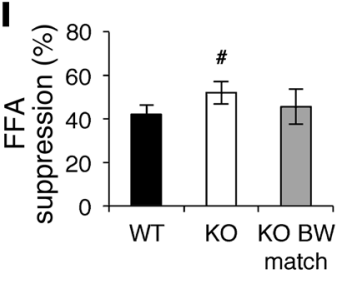

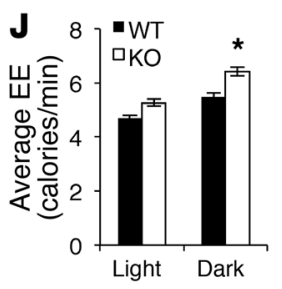
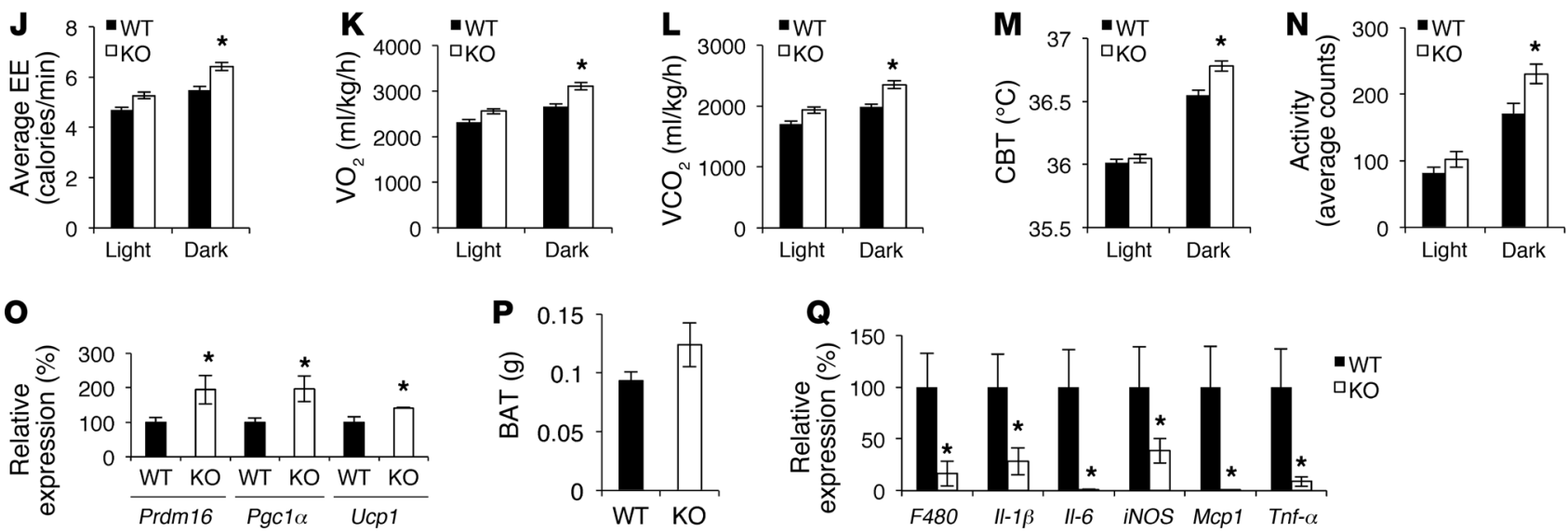

Figure 3

Effect of Gpr21 deletion on BW gain, food intake, and glucose tolerance. (A) BW gain and (B) weekly food intake of 12-week HFD-fed Gpr21 KO and WT littermates ( $n=38-40$ per group). (C) i.p. GTT $(1 \mathrm{~g} / \mathrm{kg})$ on BW-matched Gpr21 KO and WT mice fed HFD for 11 weeks $(n=5-6$ per group). (D) Insulin concentration at the indicated time points. (E-I) Hyperinsulinemic-euglycemic clamp study on Gpr21 KO and WT littermates ( $n=6$ WT, 12 Gpr21 KO, 6 Gpr21 KO BW-matched to WT). (E) GIR. (F) Basal HGP. (G) HGP suppression by insulin. (H) IS-GDR. (I) Insulininduced FFA suppression. (J) EE in Gpr21 KO and WT littermates over a 24-hour period. (K) Average VO $\mathrm{V}_{2}(\mathrm{ml} / \mathrm{kg} / \mathrm{h})$ and $(\mathbf{L})$ average VCO 2 in the light and dark cycles. (M) CBT of Gpr21 KO and WT mice in a 24-hour period, averaged across 3 days. (N) Average activity in the dark and light cycles. (O) q-PCR analysis of BAT expression of Prdm16, Pgc1 $\alpha$, and Ucp1. (P) BAT weight. (Q) q-PCR analysis of inflammatory gene expression in BAT. ${ }^{P} P<0.1$ (NS), ${ }^{*} P<0.05$ vs. WT, repeated-measures 2-way ANOVA (A-D), 2-way ANOVA (E-I), or Student's $t$ test (J-Q).

stimulated by the chemokine-rich CM. A similar result was obtained when the specific chemokines MCP-1 and leukotriene B4 (LTB4) were used individually as chemoattractants in DMEM (Figure 5E). CCR2 expression was the same in WT and KO macrophages, which suggested that reduced chemokine receptor expression did not account for the impaired chemotaxis in Gpr21 KO macrophages. CCR2 expression in the liver and adipose tissue was also similar between Gpr21 KO and WT mice (Supplemental Figure 6A). To further assess the reduced chemotactic capacity of Gpr21 KO macrophages, we performed F-actin staining after treatment with LPS or MCP-1, which showed that WT macrophages underwent cytoskeletal reorganization to form a branched morphology (Figure 5F). Such structures, which are essential for cell morphogenesis in preparation for transmigration, were absent in Gpr21 KO macrophages. The lack of response to LPS observed in Gpr21 KO macrophages was not due to differences in TLR4 expression (Supplemental Figure 6B).
These results directly showed a cell-autonomous chemotactic defect in Gpr21 KO macrophages. To determine whether the reduction in ATMs in Gpr21 $\mathrm{KO}$ mice could also be due to decreased adipocyte chemotactic signals, we cultured primary adipocytes from Gpr21 KO and WT mice and harvested the CM. WT i.p. macrophages rapidly migrated toward the CM harvested from WT adipocytes, but the CM from Gpr21 KO mice showed a trend toward attracting fewer WT i.p. macrophages (Supplemental Figure 6C).

To determine whether GPR21 also plays an important role in human monocyte chemotaxis, we conducted a similar experiment in the human monocyte-like cell line U-937 (17) using siRNA-mediated knockdown of GPR21. Human GPR21 mRNA was depleted by $53 \%$ in U-937 cells (data not shown), which resulted in a substantial (albeit not significant) $42 \%$ decrease in chemotaxis in the transwell assay $(P<0.1$; Figure $5 \mathrm{G})$. 
A

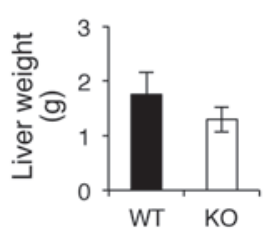

B

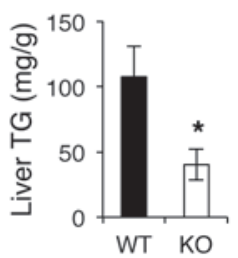

C

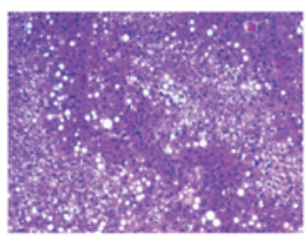

WT

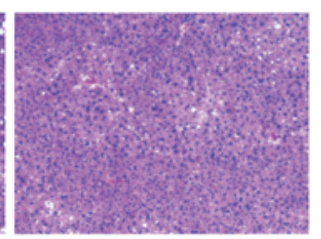

KO

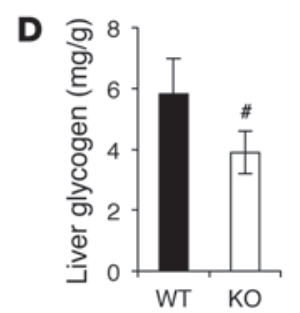

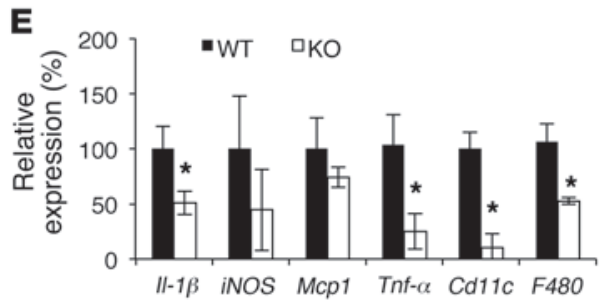

G

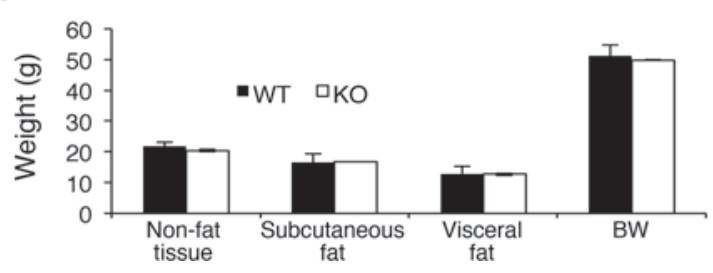

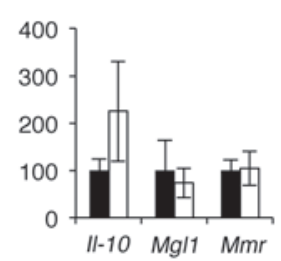

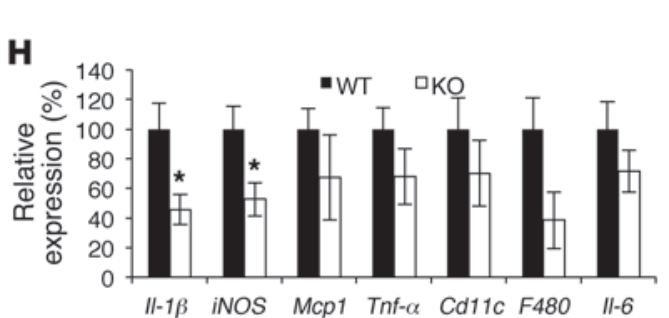

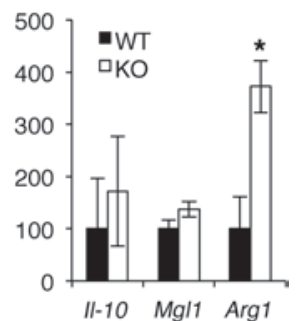

Figure 4

Inflammatory state of liver and eWAT in 12-week HFD-fed Gpr21 KO mice. (A) Liver weight from BW-matched mice. (B) Liver TG determination. (C) H\&E stain of liver sections from Gpr21 KO and WT mice. Original magnification, $\times 50$. (D) Quantitation of glycogen in the liver. (E) Inflammatory gene expression in liver. (F) eWAT weight. (G) MRI measurement of subcutaneous and visceral fat mass. (H) Inflammatory gene expression in eWAT. ${ }^{~} P<0.1$ (NS), ${ }^{*} P<0.05$ vs. WT, Student's $t$ test.

The ability of Gpr21 KO macrophages to transmigrate in vivo was assessed using a macrophage tracking technique $(18,19)$. Peripheral blood monocytes were obtained from donor Gpr21 $\mathrm{KO}$ and WT mice and labeled with fluorescent $\mathrm{PKH} 26^{+}$dye ex vivo. The labeled monocytes were then injected into obese recipient WT mice, and the number of PKH $26^{+}$cells in SVF and liver was assessed 5 days later. Significantly fewer macrophages from the Gpr21 KO mice migrated into adipose tissue $(P<0.05)$, and substantially fewer into the liver (albeit not significant; $P<0.1$ ), compared with WT cells (Figure 5, H and I).

To determine whether Gpr21 plays a role in monocyte development, we measured the levels of neuraminidase 1 (Neu1). Neu1 plays an important role in the differentiation of monocytes into macrophages, and the level of Neu1 increases as monocytes develop (20). Neu1 expression levels in monocytes, i.p. macrophages, and SVF from WT and Gpr21 KO mice were comparable (data not shown), which suggests that monocyte development is not affected by deletion of Gpr21. Furthermore, the monocyte populations of the BM and spleen were the same in Gpr21 KO and WT mice (Supplemental Figure 7, A and B).

$B M$ transplantation (BMT) studies. To more specifically assess the role of hematopoietic cells in the Gpr21 KO phenotype, we performed BMT of Gpr21 KO and WT BM into irradiated WT recipients (referred to herein as BMT-KO and BMT-WT, respectively). At 8 weeks after BMT, the groups were placed on HFD. There were no significant differences in BW or food intake between BMT-WT and BMT-KO mice fed HD (Figure 6, A and B), although it has been previously reported that BMT mice gain less weight on HFD than do nonirradiated animals. These results demonstrated that loss of Gpr21 in the hematopoietic compartment did not affect the ability of the BMT mice to become obese.

GTTs, ITTs, and hyperinsulinemic glucose clamp studies revealed that the glucose-tolerant, insulin-sensitive phenotype observed in the whole-body Gpr21 KO mouse was recapitulated in BMT-KO mice (Figure 6, C-J, and Supplemental Figure 2). Analysis of plasma inflammatory markers in HFD-fed BMT-KO mice showed significantly decreased expression of IL-6, MCP-1, and IL-1 $\beta$ (Figure 6K). The decreased proinflammatory gene expression in both eWAT and liver and increased Arg1 in liver of the Gpr21 $\mathrm{KO}$ mice was also observed in HFD-fed BMT-KO mice (Figure 6, L and M).

Gpr21 knockdown in the bypothalamus affects $B W$ and metabolic phenotype. The results of the BMT experiments in Figure 6 indicated that the majority of the metabolic phenotype in the wholebody Gpr21 KO is accounted for by the hematopoietic cell compartment, most likely macrophages. Because Gpr21 was highly expressed in the hypothalamus and preoptic area of the brain, and the whole-body Gpr21 KO mice demonstrated a decrease in BW that was not observed in BMT-KO mice, it seemed possible that the BW phenotype could be related to deletion of Gpr21 in these brain regions. To assess this possibility, we injected a lentiviral vector containing an shRNA directed against Gpr21 (lenti-shGPR21) or a nonsilencing control (lenti-control) directly into the hypothalami of WT mice; lenti-shGPR21 caused a $55 \%$ decrease in hypothalamic Gpr21 expression (Supplemen- 
A

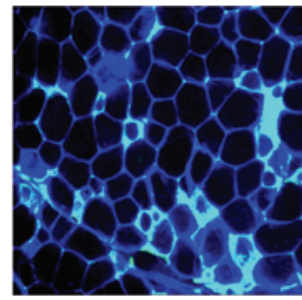

WT
KO

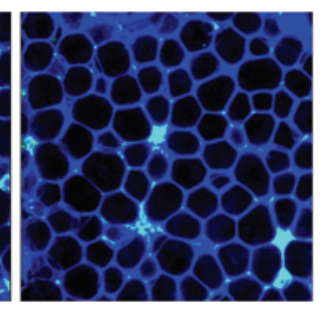

B

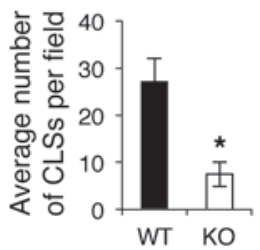

C

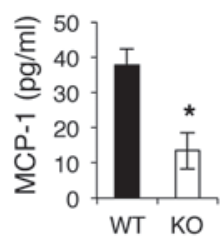

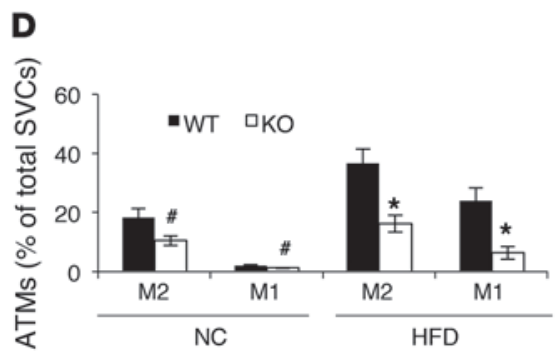

\section{E}
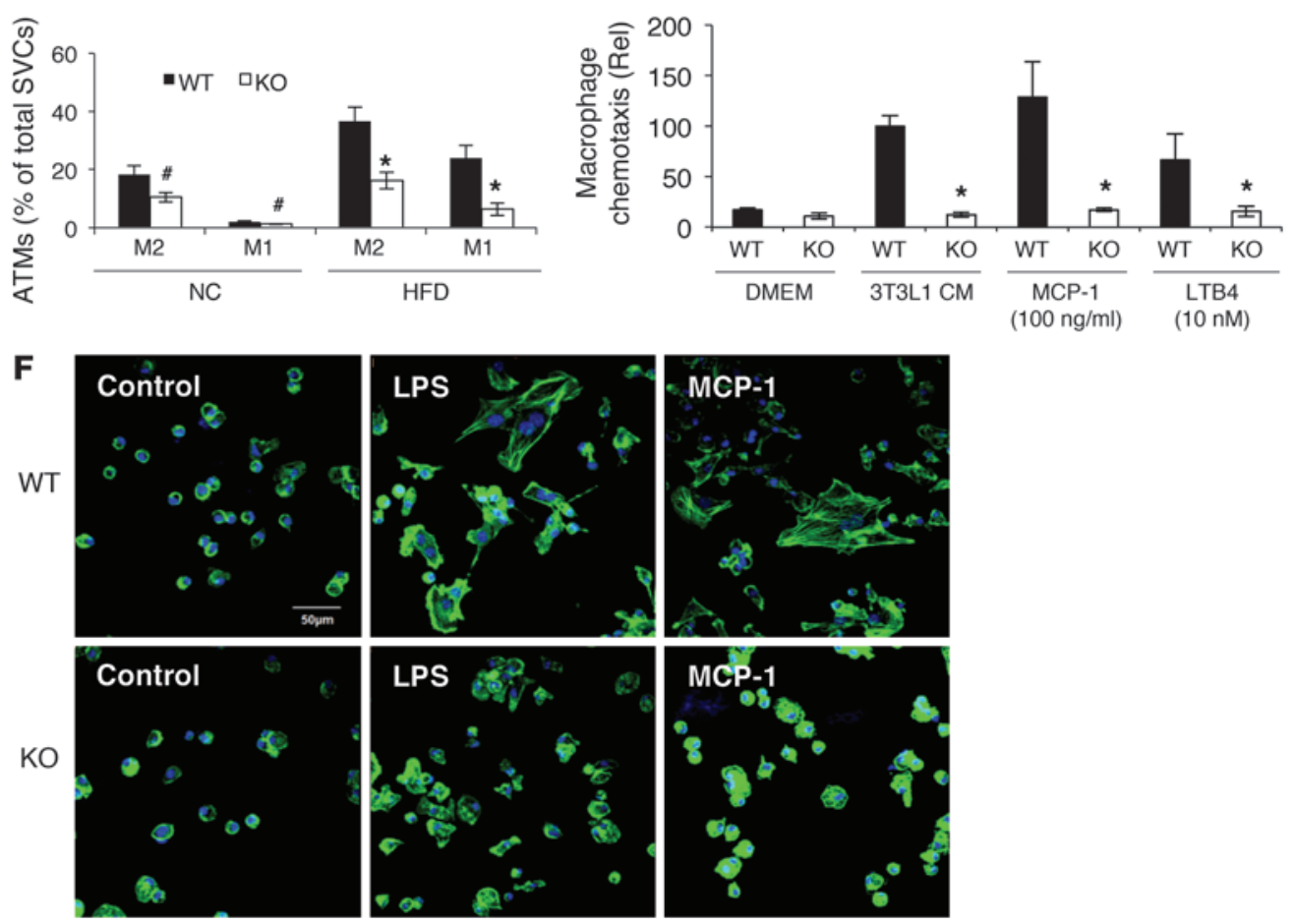

G
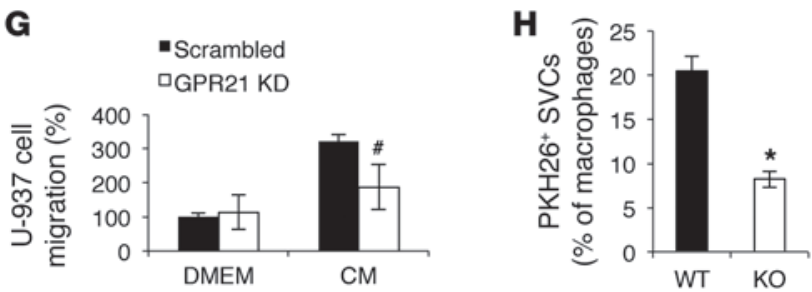

I

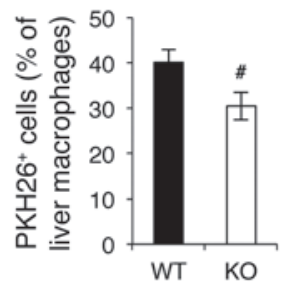

Figure 5

Decreased macrophages in adipose tissue of Gpr21 KO mice. (A) Confocal merged images from epididymal fat pads of HFD-fed Gpr21 KO and WT mice costained with anti-F4/80 (cyan) and anti-caveolin-1 (blue) antibodies. Original magnification, $\times 200$. (B) Number of crown-like structures (CLSs) per field in adipose tissue sections. (C) MCP-1 protein level in plasma of BW-matched HFDfed mice. (D) FACS analysis of M1-like and M2-like ATM subsets in stromal vascular cells (SVCs). (E) Migratory capacity of i.p. macrophages, as measured by in vitro transwell chemotaxis assay using CM from 3T3L1 adipocytes, MCP-1 (100 ng/ml), or LTB4 (10 nM). (F) Gpr21 KO macrophage exhibited dysfunctional cytoskeletal organization. i.p. macrophages were treated with LPS $(10 \mathrm{ng} / \mathrm{ml})$ or MCP-1 $(100 \mathrm{ng} / \mathrm{ml})$ for 10 minutes, and phalloidin-Alexa Fluor 488 was used to stain F-actin. Scale bar: $50 \mu \mathrm{m}$. (G) Migratory capacity of human U-937 monocytes after human GPR21 was knocked down by RNAi, measured using an in vitro transwell chemotaxis assay. ( $\mathbf{H}$ and $\mathbf{I})$ Migratory capacity of Gpr21 macrophages in vivo to eWAT (H) and liver (I), assessed by PKH26-labeled monocytes. Data are mean \pm SEM of 3 independent experiments in triplicate. ${ }^{\#} P<0.1$ (NS), ${ }^{*} P<0.05$ vs. WT. All images are representative of results from 3 independent experiments. tal Figure 8A). In the first few days after injection, both lentishGPR21- and lenti-control-injected mice experienced a reduction in BW due to the recovery from anesthesia, which quickly reversed in lenti-control-injected mice, in contrast to lentishGPR21-injected mice. Thus, there was a sustained decrease in BW in the lenti-shGPR21-injected mice, with no change in food intake compared with controls (Supplemental Figure 8, B and C). With respect to glucose homeostasis, there was a small decrease in fasting glucose levels in the lenti-shGPR21 group (WT, $117 \mathrm{mg} / \mathrm{dl}$; Gpr21 KO, $83 \mathrm{mg} / \mathrm{dl}$ ), but no change in either glucose or insulin tolerance (Supplemental Figure 8, D and E). Furthermore, no changes in circulating cytokines/chemokines were observed between the groups (Supplemental Figure 8F). In addition, Ucp 1 expression was increased in Gpr21 KO mouse
BAT (Supplemental Figure 8G), consistent with increased EE. In HFD-fed obese mice, lenti-shGPR2 1 treatment did not result in any significant effects on BW, glucose tolerance, or insulin sensitivity (Supplemental Figure 9).

\section{Discussion}

It is well known that obesity-induced inflammation is a key component of systemic insulin resistance, and increased accumulation of proinflammatory macrophages in adipose tissue and liver is a major driver of this inflammation/insulin resistance syndrome. Thus, both obesity and inflammation emerge as key etiologic factors in insulin-resistant states, including type 2 diabetes mellitus. Here we showed that the orphan GPCR GPR21 was highly expressed in the hypothalamus and macrophages in mice and 
A

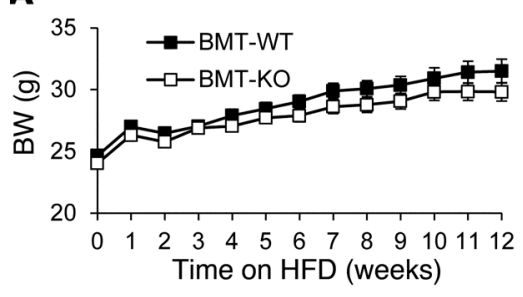

B

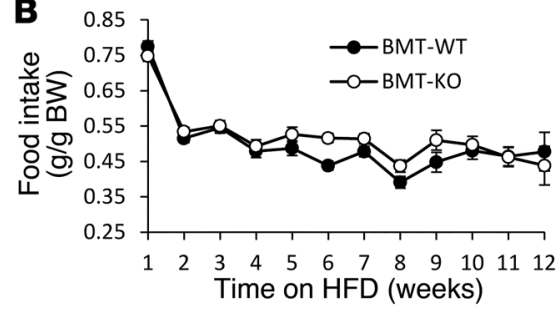

C

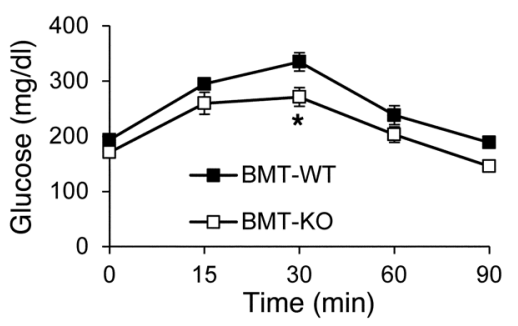

D

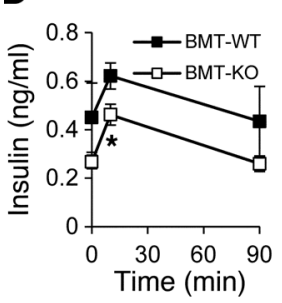

E

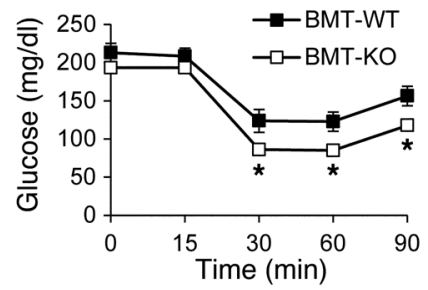

F

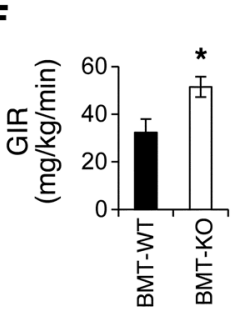

G

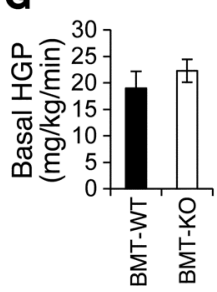

I

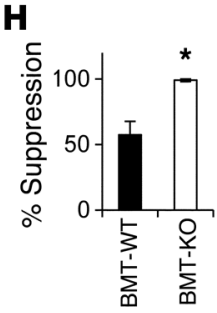

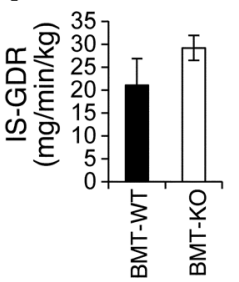
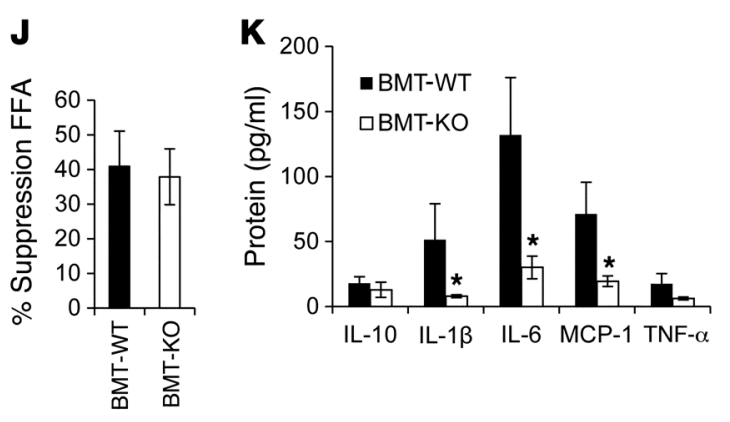

L $\stackrel{\overbrace{}}{\stackrel{0}{\varrho}} 300$
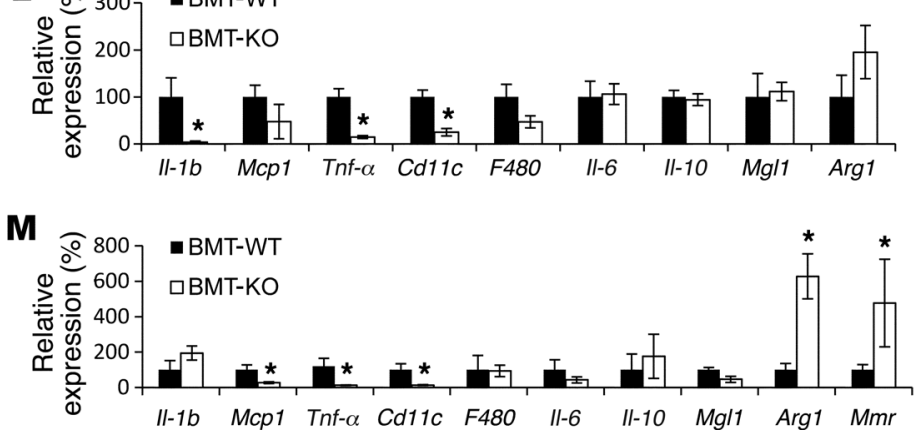

Figure 6

BMT studies. (A) BW gain and (B) daily food intake of 12-week HFD-fed BMT-WT and BMT-KO mice. (C) GTT ( $n=9-11$ mice per group). (D) Insulin concentration. (E) ITT. (F-J) Hyperinsulinemic-euglycemic clamp studies in BMT-KO and BMT-WT littermates $(n=5-6$ per group). (F) GIR. (G) Basal HGP. (H) HGP suppression by insulin. (I) IS-GDR. (J) Insulin-stimulated FFA suppression. (K) Plasma protein expression of inflammatory markers. q-PCR analysis of inflammatory gene expression in (L) eWAT and (M) liver. ${ }^{*} P<0.05$ vs. WT, repeated-measures 2 -way ANOVA (A-E) or Student's $t$ test $(\mathbf{F}-\mathbf{I})$.

that whole-body $\mathrm{KO}$ of this receptor led to robust improvement in glucose tolerance, insulinemia, and systemic insulin sensitivity as well as a modest lean phenotype. The improvement in insulin sensitivity was traced to a marked reduction in tissue inflammation due to decreased chemotaxis of Gpr $21 \mathrm{KO}$ macrophages, with reduced ATM content in adipose tissue and liver of HFD-fed Gpr21 KO compared with WT mice. Our data suggest that GPR21 may be a novel control point coordinating macrophage proinflammatory activity in the context of obesity-induced insulin resistance.

In this study, Gpr21 deficiency resulted in decreased BW (on average $\sim 4 \mathrm{~g}$ ) in mice fed NC. On HFD, BW gain was the same between these genotypes, but since Gpr21 KO mice started out approximately $4 \mathrm{~g}$ lighter, the absolute final BW of Gpr $21 \mathrm{KO}$ mice was somewhat less than that of WT mice. However, we studied a large cohort of HFD-fed mice, with a range in BW, and this enabled us to BW-match a group of HFD-fed Gpr21 KO mice with agematched WT mice, segregating out the effects of Gpr21 deficiency without the confounding influence of BW differences. This is a particularly important point in the context of insulin resistance and glucose homeostasis, as changes in BW influence insulin sensitivity (21). We found that BW-matched HFD-fed Gpr21 KO mice still maintained improved glucose tolerance and systemic insulin sensitivity. Hyperinsulinemic clamp studies and ex vivo measurements of insulin action revealed that BW-matched Gpr $21 \mathrm{KO}$ mice displayed improved systemic insulin sensitivity that was primarily manifested in liver and adipose tissue.

We found marked decreases in inflammatory gene expression in adipose tissue and liver in HFD-fed Gpr $21 \mathrm{KO}$ versus WT mice, and it is well known that obesity leads to infiltration of large numbers of inflammatory macrophages into adipose tissue $(5,22)$ and liver (6). To quantitate differences in ATMs, we performed immunohistological staining for the macrophage marker F4/80, and found markedly fewer macrophages in Gpr21 KO than in WT eWAT. In vitro analysis of macrophage chemotaxis showed that Gpr21 KO macrophages display defective chemotactic activity in response to adipocyte CM or MCP-1. The finding that Gpr21 KO macrophages also did not migrate toward the adipocyte $\mathrm{CM}$ raises the possibility that the ligand for Gpr 21 could be expressed in the media. However, the finding that Gpr21 KO macrophages also did not migrate toward the chemokine MCP-1 (which binds to the CCR2 receptor, which is normally expressed on Gpr21 KO macrophages) suggested there may be a more generalized defect in the migration ability of 
Gpr21 KO macrophages. We therefore examined the cytoskeletal organization of Gpr $21 \mathrm{KO}$ and WT macrophages in response to LPS proinflammatory stimulation, and found that Gpr21 KO macrophages did not undergo cytoskeletal reorganization to form the branched morphology essential for cell morphogenesis in preparation for transmigration. This defect in the migratory capacity of Gpr $21 \mathrm{KO}$ macrophages was confirmed in vivo by injecting and tracking PKH26 fluorescent dye-labeled Gpr21 KO and WT monocytes into WT HFD-fed obese mice, measuring the number of labeled macrophages that appeared in the liver and adipose tissue. As predicted, fewer Gpr $21 \mathrm{KO}$ monocytes migrated to the adipose tissue and liver compared with WT cells, fully consistent with the chemotaxis of Gpr $21 \mathrm{KO}$ macrophages observed in vitro. To determine whether the defect in murine macrophage chemotaxis is relevant to human cells, we performed a similar in vitro assay using U-937 human monocyte-like cells and found that knockdown of human GPR 21 by $53 \%$ resulted in a substantial decrease in the number of cells that migrated toward CM.

To further delineate the role of macrophage deletion of Gpr21 in the improved insulin sensitivity and decreased inflammation in whole-body Gpr21 KO mice, we performed adoptive BMT into WT recipients, generating BMT-KO and BMT-WT chimeras. Absence of Gpr21 in the hematopoietic compartment led to improved glucose tolerance and systemic insulin sensitivity, and hyperinsulinemic clamp studies revealed enhanced insulin sensitivity, consistent with the results in the whole-body Gpr $21 \mathrm{KO}$ mice. In common with the whole-body Gpr21 KO, hematopoietic Gpr21 KO also resulted in decreased proinflammatory gene expression in the adipose tissue and liver. Thus, the phenotype observed in the hematopoietic Gpr21 KO mice largely resembled the whole-body Gpr $21 \mathrm{KO}$ phenotype, which indicates that macrophage deletion of Gpr 21 is the dominant cause of the metabolic aspects of the phenotype.

Our results clearly showed that Gpr21 deletion in macrophages substantially compromised the ability of those cells to undergo migration toward the chemokine-enriched CM harvested from the adipocytes or toward MCP-1 in buffer and to undergo in vivo migration into adipose tissue and liver. This was confirmed by FACS analysis of the SVF, which showed decreased numbers of both M1-like and M2-like ATMs in Gpr21 KO mice fed either NC or HFD. The reduction in M1-like ATMs therefore provides a reasonable mechanism for the decreased tissue inflammation and improved insulin sensitivity observed in HFD-fed obese Gpr21 KO mice. In lean mice, the proportion of M2-like ATMs was greater than in obese mice, and recent studies indicate that resident alternatively activated M2-like ATMs have a greater propensity to proliferate than do M1-like ATMs (23). Whether the reduced number of M2-like ATMs in Gpr21 $\mathrm{KO}$ mice is indicative of a defect in this proliferative process is unknown. Gpr21 KO appears to affect more than a single step in the process of monocytes becoming ATMs, including impairing the ability of monocytes to transmigrate into tissues and of mature macrophages to migrate toward chemotactic signals.

The signaling mechanisms whereby GPR 21 mediates these effects will be of great interest. However, almost nothing is known about GPR21 signaling, other that it may be coupled to Gq. Since natural ligands for GPR21 are unknown and there are no small molecules available that interact with this receptor, further definitive studies on GPR21 signaling are severely hampered. Once agonists and antagonists of GPR21 become available, it will be of great interest to study the precise signaling mechanisms in which this receptor engages to modulate macrophage chemotaxis.
We found Gpr21 to be highly expressed in the hypothalamus, in which peripheral signals and neural pathways are integrated to control energy homeostasis and BW. The hypothalamus is composed of many nuclei that perform distinct functions, including the preoptic area, which controls CBT regulation (24-26). Gpr21 was highly expressed in the preoptic area, which suggests that this receptor may play a role in $\mathrm{CBT}$ and energy homeostasis. Indeed, Gpr21 KO mice displayed increased EE and CBT compared with their WT littermates primarily in the dark cycle, largely due to increased activity. To determine whether the increased EE in Gpr21 KO mice was exclusively the result of increased physical activity or whether there was also a difference in thermogenesis, we analyzed BAT. BAT was increased in Gpr21 KO mice and also exhibited enhanced mRNA expression of Ucp1 (which is important in generation of heat by nonshivering thermogenesis) and of Prdm16 and Pgc1 $\alpha$, markers consistent with BAT expansion. We exposed the mice to a cold challenge and found that Gpr21 $\mathrm{KO}$ mice had a greater ability to maintain CBT by increasing BAT activity to generate heat.

To more specifically asses the effect of hypothalamic Gpr21 on BW, we depleted Gpr21 by injecting lenti-shGPR21 into the hypothalamus. This led to decreased BW with NC feeding, but not with HFD feeding, precisely what we observed in whole-body Gpr $21 \mathrm{KO}$ mice. We conclude that the magnitude of the effect of hypothalamic Gpr21 depletion on BW is modest and masked by either the HFD or the resulting HFD-induced obesity. Having said this, the EE required to maintain $\mathrm{CBT}$ is a large component of total daily $\mathrm{EE}$, and subtle changes in CBT could have more significant effects on BW over extended periods of time (27).

In summary, we showed that Gpr21 played an important role in the migration of macrophages into inflammatory tissue in obesity and that macrophage-specific deletion of this receptor improved obesity-induced inflammation and insulin sensitivity. Results of in vitro chemotaxis studies in human monocytes suggested that the defect in chemotaxis observed ex vivo and in vivo in mice is also translatable to humans. Hypothalamic expression of Gpr21 also contributed to EE, and depletion of Gpr21 in the hypothalamus resulted in decreased BW, but this effect was comparatively small. These results suggest that pharmacological GPR21 inhibition could protect against the negative effects of HFD by increasing EE and improving insulin sensitivity.

\section{Methods}

Further information can be found in Supplemental Methods.

Mice. Gpr21 KO ES cells were obtained from Deltagen. These ES cells contain an entire deletion of exon 1 of Gpr21, which is replaced by a 5.3-kb LacZ/ Neo cassette in the Gpr21 targeting vector. Gpr $21 \mathrm{KO}$ mice were generated by the Scripps Research Institute mouse Genetics Core. We backcrossed Gpr21 $\mathrm{KO}$ mice onto a C57BL/ 6 background for more than 6 generations. Only male mice were used for experiments. Mice were housed in a 12-hour light, 12-hour dark cycle. Beginning at 8 weeks of age, mice were either fed NC (13.5\% fat; LabDiet) or HFD (60\% fat; D12492, Research Diets) ad libitum for up to 20 weeks, and BW and food intake was monitored. For genotyping, forward primer 5'-ATACAGAGGCGTAGTCTCCAGGGAG-3' was used, in combination with reverse primer 5'-AGTGACAGTAGCTGCTCCTGAGAAC-3' to detect endogenous Gpr21 or 5'-GGGCCAGCTCATTCCTCCCACTCAT-3' to detect mutant Gpr21 mRNA.

Metabolic studies. GTTs allowed us to determine the effectiveness of insulin in reducing fasting glucose levels. For GTTs, an i.p. dose of $1 \mathrm{~g} / \mathrm{kg}$ dextrose was administered. Blood insulin concentration was measured before 
glucose injection (after 6 hours of fasting) as well as 10 and 90 minutes after injection. For ITTs, an i.p. dose of $0.35 \mathrm{U} / \mathrm{kg}$ insulin was administered, and blood glucose was measured at the indicated time points.

We performed hyperinsulinemic-euglycemic clamp studies as previously described (18). Briefly, dual catheters (catalog no. MER-025; Braintree Scientific) were implanted in the right jugular vein, tunneled subcutaneously, and exteriorized at the back of the neck. Mice were allowed to recover for 3 days prior to the clamp procedure. After a 6-hour fast, blood glucose was assessed via tail nick, $\mathrm{BW}$ was measured, and mice were placed in a Lucite restrainer (Braintree Scientific). A constant infusion of D- $\left[3-{ }^{3} \mathrm{H}\right]$ glucose $(5 \mu \mathrm{Ci} / \mathrm{h}$; DuPont-NEN) was administered. After 90 minutes of tracer equilibration and basal sampling at -10 and 0 minutes, glucose $(50 \%$ dextrose, variable infusion; Abbott) and tracer $(5 \mu \mathrm{Ci} / \mathrm{h})$ plus insulin $(6 \mathrm{mU} / \mathrm{kg} / \mathrm{min})$ was infused via the jugular vein cannulae. Blood was sampled from tail clips at 10-minute intervals and analyzed for glucose. Steady-state conditions (120 $\pm 10 \mathrm{mg} / \mathrm{dl}$ ) were achieved at the end of the clamp by maintaining glucose infusion and plasma glucose concentration for a minimum of 30 minutes. Blood samples were taken at $-10,0$ (basal), 110, and 120 (end of experiment) minutes to determine glucose-specific activity and insulin and FFA levels. Stress was minimized during the clamp procedure. Mice were familiar with the experimenter, as they had been handled frequently prior to the study. The room in which clamp experiments were performed was quiet with subdued lighting and controlled temperature, and by visual observation, the mice appeared to acclimatize well to the experimental conditions. After the procedure, mice were returned to their cages immediately and allowed to fully recover. We quantified tracer-determined rates by using the Steele equation for steady-state conditions (28). HGP and GDR were calculated in the basal state and during the steady-state portion of the clamp. At steady state, the rate of glucose disappearance (i.e., total GDR) is equal to the sum of the rate of endogenous glucose production (i.e., HGP) plus the exogenous (cold) GIR. The IS-GDR is equal to the total GDR minus the basal glucose turnover rate. To asses the biochemical response to insulin stimulation in vivo, we performed acute insulin challenge experiments (see Supplemental Methods).

Measurement of CBT and activity. Measurement of CBT and locomotor activity was performed as previously described (13). In brief, radiotelemetry devices were implanted into the i.p. cavity, and CBT and activity was recorded every 15 minutes over a 24 -hour period. To asses the CBT of Gpr21 KO and WT mice when exposed to cold challenge, we performed cold tolerance tests (see Supplemental Methods).

Indirect calorimetry. Mice were placed into Comprehensive Lab Animal Monitoring System (CLAMS; Columbus Instruments) metabolic cages to adapt to their surroundings for 48 hours before study. Rates of $\mathrm{O}_{2}$ consumption $\left(\mathrm{VO}_{2} ; \mathrm{ml} / \mathrm{kg} / \mathrm{h}\right)$ and $\mathrm{CO}_{2}$ production $\left(\mathrm{VCO}_{2}\right)$ were measured for each chamber every 17 minutes throughout the study. EE was calculated as $\mathrm{VO}_{2} \times\left(3.815+\left[1.232 \times\left(\mathrm{VCO}_{2} / \mathrm{VO}_{2}\right)\right]\right)$.

MRI. After 16 weeks of HFD feeding, lean body mass and fat pad volumes were measured by MRI as previously described (29).

RNA isolation, semiquantitative RT-PCR, and $q-P C R$. Total RNA was isolated using TRIzOL (Invitrogen) according to the manufacturer's instructions. First-strand cDNA was synthesized using a High-Capacity cDNA Reverse Transcription Kit (Applied Biosystems). For q-PCR, samples were run in a 20- $\mu$ l reaction (iTaq SYBRgreen supermix; BioRad) using a stepOnePlus Real-Time PCR system (Applied Biosystems). Gene expression levels were calculated after normalization to the standard housekeeping gene Actb and RNA polymerase II, using the $\Delta \Delta$ Ct method as described previously (30), and expressed as relative mRNA levels compared with internal control. See Supplemental Table 1 for primers.

ELISA. IL-10, IL-1 $\beta$, IL-6, MCP-1, and TNF- $\alpha$ serum levels were measured using multiplex ELISA (Millipore/Linco Research). Plasma insulin levels were measured by ELISA (ALPCO).
Plasma FFA levels were measured enzymatically using a commercially available kit (NEFA C; Wako Chemicals). TG levels were measured enzymatically using a commercially available kit (L-Type TG M; Wako Chemicals). Glycogen levels were determined using a glycogen assay kit (KA0861; Abnova).

Confocal microscopy of mouse adipose tissue. Mice were euthanized, and fingernail-sized fat pad samples were excised and blocked for 1 hour using $5 \%$ BSA in PBS with gentle rocking at room temperature. For detection of intracellular antigens, blocking and subsequent incubations were done using 5\% BSA in PBS with 0.3\% Triton X-100. Primary antibodies were diluted in blocking buffer to $0.5-1 \mu \mathrm{g} / \mathrm{ml}$ and added to fat samples overnight at $4^{\circ} \mathrm{C}$. After 3 washes, fluorochrome-conjugated secondary antibodies were added for 1 hour at room temperature. Fat pads were imaged on an inverted confocal microscope (Fluoview 1000; Olympus). Anti-mouse antibodies used were against F4/80 (Abcam) and caveolin-1 (BD Biosciences). Quantitation of crown-like structures in WT and Gpr21 KO mice was done by counting the number of crown-like structures per field for a minimum of 6 mice per group.

In vitro chemotaxis assay. In vitro chemotaxis assay was performed as previously described (31). Briefly, i.p. macrophages were harvested from WT and Gpr21 KO mice as described previously (32). Mature 3T3-L1 adipocytes showing large lipid droplets (12 days after differentiation protocol initiation) were used for preparation of $\mathrm{CM}$. In the migration experiment, 100,000 i.p. macrophages from WT or Gpr21 KO mice were used per condition. The i.p. macrophages were placed in the upper chamber of an 8 - $\mu \mathrm{m}$ polycarbonate filter (24-transwell format; Corning), and adipocyte CM, MCP-1 (100 ng/ml; R\&D Systems), or LTB4 (Cayman Chemical) was placed in the lower chamber. After 3 hours of migration, cells were fixed in formalin and stained with DAPI for observation.

The same procedure was followed for the human chemotaxis experiments, except human U-937 monocyte-like cells (a gift from C. Glass, UCSD, La Jolla, California, USA) were used in place of i.p. macrophages. In brief, U-937 cells in 60-mm culture dishes were transfected using Lipofectamine RNAiMAX transfection reagent (Invitrogen) with 10 pmol RNAi to knock down the expression of Gpr21 (catalog no. L-005556-00; Thermo Scientific) or with scrambled human RNAi (Thermo Scientific) for 6 hours in serum-free DMEM.

To generate $\mathrm{CM}$ from murine primary cultured adipocytes, adipose tissue was collected from WT and Gpr21 KO mice, and adipocytes were extracted and cultured overnight as previously described (32). Briefly, CM was collected and stored at $-20^{\circ} \mathrm{C}$ for use in the macrophage tracking experiments.

F-actin staining. i.p. macrophages were plated on sterile coverslips placed in 24-well plates and incubated overnight at $37^{\circ} \mathrm{C}$ in a $\mathrm{CO}_{2}$ incubator to form a monolayer. We treated i.p. macrophages with LPS $(10 \mathrm{ng} / \mathrm{ml})$, MCP-1 (100 ng/ml; R\&D Systems), or medium alone (serum free) for 10 minutes. After washing, cells were fixed in $4 \%$ PFA for 10 minutes, then washed twice in PBS and permeabilized with $0.1 \%$ Triton X-100 for 5 minutes. Cells were washed again twice in PBS, incubated in PBS containing 1\% BSA for 10 minutes, then incubated in phalloidin-FITC solution $(500 \mathrm{nM}$; Invitrogen) for 20 minutes to stain F-actin. The coverslips were washed twice in PBS and mounted on slides for microscopy.

In vivo macrophage tracking. In vivo macrophage tracking was performed as described previously $(18,19)$. In brief, leukocytes were prepared from C57BL/6 male mice, and monocyte subsets were enriched with EasySep Mouse Monocyte Enrichment Kit (STEMCELL Technologies) according to the manufacturer's instructions. Isolated monocytes $\left(5-10 \times 10^{6}\right)$ were labeled with PKH26 fluorescent dye. Approximately $1 \times 10^{6}$ viable labeled cells were injected into the femoral vein of each mouse group; 5 days after injection, ATMs were immediately isolated from visceral fat tissue and analyzed by FACS. 
FACS analyses. Epididymal fat pads were weighed, rinsed in PBS, and minced in FACS buffer (PBS containing 1\% BSA). Adipocytes and stromal vascular cells were prepared from collagenase-digested adipose tissue. FACS analysis of stromal vascular cells for macrophage content were performed as previously described (33). T cells were obtained using an EasySep Mouse T Cell Enrichment kit (STEMCELL Technologies) according to the manufacturer's protocol.

$B M T$. We performed BMT experiments to ascertain the role of macrophage Gpr21 in mediating whole-body glucose homeostasis. BMT was performed as described previously (34). In brief, BM obtained from WT and Gpr $21 \mathrm{KO}$ mice $\left(\sim 3 \times 10^{6}\right.$ cells) was injected via the tail vein into 8-weekold male irradiated (10 Gy) C57BL/ 6 mice. Mice were allowed 8 weeks for reconstitution of donor BM (verified by $\mathrm{q}-\mathrm{PCR}$ ), and then animals were fed either NC or HFD for 20 weeks, during which GTT, ITT, and clamps were performed.

Chemicals and reagents. All chemicals were purchased from Sigma-Aldrich unless otherwise indicated.

Relative $\beta$ cell area determination. As previously described, $\beta$ cell area was calculated as insulin-positive area relative to total pancreatic area (35). See Supplemental Methods for details.

Statistics. All statistical analysis was performed by 2-tailed Student's $t$ test using Excel (Microsoft), or by 2 -way ANOVA (with repeated measures where necessary) followed by Bonferroni's post-hoc test using GraphPad Prism version 5. A $P$ value less than 0.05 was considered significant. All data are expressed as mean \pm SEM.

1. Lumeng CN, Saltiel AR. Inflammatory links between obesity and metabolic disease. J Clin Invest. 2011;121(6):2111-2117.

2. Olefsky JM, Glass CK. Macrophages, inflammation, and insulin resistance. Annu Rev Physiol. 2010;72:219-246.

3. Schenk S, Saberi M, Olefsky JM. Insulin sensitivity: modulation by nutrients and inflammation. J Clin Invest. 2008;118(9):2992-3002.

4. Weisberg SP, et al. CCR2 modulates inflammatory and metabolic effects of high-fat feeding. J Clin Invest. 2006;116(1):115-124.

5. Xu H, et al. Chronic inflammation in fat plays a crucial role in the development of obesity-related insulin resistance. J Clin Invest. 2003;112(12):1821-1830.

6. Obstfeld AE, et al. C-C chemokine receptor 2 (CCR2) regulates the hepatic recruitment of myeloid cells that promote obesity-induced hepatic steatosis. Diabetes. 2010;59(4):916-925.

7. Cai D, et al. Local and systemic insulin resistance resulting from hepatic activation of IKK-beta and NF-kappaB. Nat Med. 2005;11(2):183-190.

8. Arkan MC, et al. IKK-beta links inflammation to obesity-induced insulin resistance. Nat Med. 2005;11(2):191-198.

9. Lanthier N, Horsmans Y, Leclercq IA. Clodronate liposomes: all sites of injection are not equal. Hepatology. 2010;51(2):721-722.

10. Neyrinck AM, Cani PD, Dewulf EM, De Backer F, Bindels LB, Delzenne NM. Critical role of Kupffer cells in the management of diet-induced diabetes and obesity. Biochem Biophys Res Commun. 2009;385(3):351-356

11. Eberwine J, Bartfai T. Single cell transcriptomics of hypothalamic warm sensitive neurons that control core body temperature and fever response Signaling asymmetry and an extension of chemical neuroanatomy. Pharmacol Ther. 2011;129(3):241-259.

12. Osborn $\mathrm{O}$, et al. $\mathrm{Ccl} 22 / \mathrm{MDC}$, is a prostaglandin dependent pyrogen, acting in the anterior hypothalamus to induce hyperthermia via activation of brown adipose tissue. Cytokine. 2011;53(3):311-319.
Study approval. All procedures were approved by the UCSD animal care and use committee.

\section{Acknowledgments}

We thank Elizabeth J. Hansen for editorial assistance and the UCLA DERC CORE for ELISA measurements. We thank the TSRI mouse genetic core for mouse line generation, the Flow Cytometry Resource and Neal Sekiya (VA San Diego Hospital, San Diego, California) for assistance with FACS analysis, and UCSD Neuroscience Microscopy (supported by shared facility grant P30 NS047101) for microscopy analysis. This study was funded in part by NIH grants NIDDK DK033651, DK063491, and DK074868 to J.M. Olefsky; by EU Ideas program grant ERC-2008-AdG-23118; by the Swiss National Science Foundation; and by the Eunice Kennedy Shriver NICHD/NIH (through cooperative agreement U54 HD 012303-25, as part of the specialized Cooperative Centers Program in Reproduction and Infertility Research).

Received for publication November 15, 2011, and accepted in revised form April 24, 2012.

Address correspondence to: Jerrold M. Olefsky, Department of Medicine, University of California, San Diego, 9500 Gilman Drive, La Jolla, California 92093-0673, USA. Phone: 858.534.6651; Fax: 858.534.6653; E-mail: jolefsky@ucsd.edu.
13. Sanchez-Alavez $M$, et al. Insulin-like growth factor 1-mediated hyperthermia involves anterior hypothalamic insulin receptors. J Biol Chem. 2011;286(17):14983-14990.

14. Sanchez-Alavez M, et al. Insulin causes hyperthermia by direct inhibition of warm-sensitive neurons. Diabetes. 2010;59(1):43-50.

15. Vassilatis DK, et al. The $G$ protein-coupled receptor repertoires of human and mouse. Proc Natl Acad Sci US A. 2003;100(8):4903-4908.

16. Bresnick JN, et al. Identification of signal transduction pathways used by orphan g proteincoupled receptors. Assay Drug Dev Technol. 2003; 1(2):239-249.

17. Minta JO, Pambrun L. In vitro induction of cytologic and functional differentiation of the immature human monocytelike cell line U-937 with phorbol myristate acetate. Am J Pathol. 1985;119(1):111-126.

18. Li P, et al. Adipocyte NCoR knockout decreases PPARgamma phosphorylation and enhances PPARgamma activity and insulin sensitivity. Cell. 2011;147(4):815-826.

19. Oh da Y, Morinaga H, Talukdar S, Bae EJ, Olefsky $\mathrm{JM}$. Increased macrophage migration into adipose tissue in obese mice. Diabetes. 2012;61(2):346-354.

20. Stamatos NM, et al. Differential expression of endogenous sialidases of human monocytes during cellular differentiation into macrophages. FEBS J. 2005;272(10):2545-2556.

21. Lee YS, et al. Inflammation is necessary for longterm but not short-term high-fat diet-induced insulin resistance. Diabetes. 2011;60(10):2474-2483.

22. Weisberg SP, McCann D, Desai M, Rosenbaum M, Leibel RL, Ferrante AW Jr. Obesity is associated with macrophage accumulation in adipose tissue. J Clin Invest. 2003;112(12):1796-1808.

23. Jenkins SJ, et al. Local macrophage proliferation, rather than recruitment from the blood, is a signature of TH2 inflammation. Science. 2011;332(6035):1284-1288.

24. Williams G, Harrold JA, Cutler DJ. The hypothalamus and the regulation of energy homeo- stasis: lifting the lid on a black box. Proc Nutr Soc. 2000;59(3):385-396.

25. Nakamura K, Morrison SF. A thermosensory pathway that controls body temperature. Nat Neurosci. 2008;11(1):62-71.

26. Nakayama T, Eisenman JS, Hardy JD. Single unit activity of anterior hypothalamus during local heating. Science. 1961;134(3478):560-561.

27. Landsberg L, Young JB, Leonard WR, Linsenmeier RA, Turek FW. Is obesity associated with lower body temperatures? Core temperature: a forgotten variable in energy balance. Metabolism. 2009;58(6):871-876.

28. Steele R. Influences of glucose loading and of injected insulin on hepatic glucose output. Ann NY Acad Sci. 1959;82:420-430.

29. Solinas G, et al. JNK1 in hematopoietically derived cells contributes to diet-induced inflammation and insulin resistance without affecting obesity. Cell Metab. 2007;6(5):386-397.

30. Yoshizaki T, et al. SIRT1 exerts anti-inflammatory effects and improves insulin sensitivity in adipocytes. Mol Cell Biol. 2009;29(5):1363-1374.

31. Patsouris D, Neels JG, Fan W, Li PP, Nguyen MT, Olefsky JM. Glucocorticoids and thiazolidinediones interfere with adipocyte-mediated macrophage chemotaxis and recruitment. J Biol Chem. 2009;284(45):31223-31235

32. Oh DY, et al. GPR120 is an omega-3 fatty acid receptor mediating potent anti-inflammatory and insulin-sensitizing effects. Cell. 2010;142(5):687-698.

33. Nguyen MT, et al. A subpopulation of macrophages infiltrates hypertrophic adipose tissue and is activated by free fatty acids via Toll-like receptors 2 and 4 and JNK-dependent pathways. J Biol Chem. 2007;282(48):35279-35292.

34. Lesniewski LA, et al. Bone marrow-specific Cap gene deletion protects against high-fat diet-induced insulin resistance. Nat Med. 2007;13(4):455-462.

35. Kavaler S, et al. Pancreatic beta-cell failure in obese mice with human-like CMP-Neu5Ac hydroxylase deficiency. FASEB J. 2011;25(6):1887-1893. 